\title{
RNAi and double-strand RNA
}

\author{
Phillip A. Sharp ${ }^{1}$ \\ Center for Cancer Research and Department of Biology, Massachusetts Institute of Technology, \\ Cambridge, Massachusetts 02139-4307 USA
}

Double-strand RNA (dsRNA) is a signal for gene-specific silencing of expression in a number of organisms. This phenomenon was demonstrated recently in Caenorhabditis elegans when dsRNA was injected into the worm and the corresponding gene products disappeared from both the somatic cells of the organism as well as in its $F_{1}$ progeny (Fire et al. 1998). This RNA interference, RNAi, has been generalized to many genes in C. elegans (Montgomery and Fire 1998; Shi and Mello 1998; Tabara et al. 1998; Timmons and Fire 1998). ds-RNA can also suppress expression of specific genes in plants, a component of the phenomenon called cosuppression (Vionnet et al. 1998; Waterhouse et al. 1998). Two recent reports document dsRNA-mediated interference with expression of specific genes in other organisms. Double-strand RNA produced gene-specific phenotypes in Trypanosoma brucei (Ngo et al. 1998) and, very recently, dsRNA-mediated interference was demonstrated in Drosophila (Kennerdell and Carthew 1998). Thus, the RNAi phenomenon is likely to be a general mechanism for gene regulation and may be critical for many developmental and antiviral processes.

Montgomery et al. (1998) have investigated how RNAi suppresses the expression of endogenous genes in C. elegans. dsRNA might conceivably direct mutations of the endogenous genes thus inactivating function. However, the fact that the $\mathrm{F}_{2}$ progeny from RNAi-treated C. elegans generally reverted to normal phenotype argued against nonreversible gene modification. Further sequencing of the targeted locus failed to detect nucleotide differences, direct evidence against a mutational mechanism.

Double-strand RNA primarily suppresses gene expression by a post-transcriptional mechanism in C. elegans (Montgomery et al. 1998). A post-transcriptional mechanism was foreshadowed by earlier experiments showing that dsRNAs from sequences in the mature RNA, that is, exons, had RNAi activity, whereas dsRNAs from intron sequences did not (Fire et al. 1998). The most direct evidence for a post-transcriptional effect arises from analysis of RNAi effects on a multiple-gene operon. Such operons are expressed in C. elegans by transcription of long precursor RNAs that are then processed by trans-splicing and cleavage to generate specific mRNAs. The lin-15b

${ }^{1}$ E-MAIL sharppa@mit.edu; FAX (617) 253-3867. and $\operatorname{lin}-15 a$ genes are part of one operon and both need to be inactivated to generate the multivulva phenotype. Injection of dsRNA from both genes generated the phenotype, whereas injection of dsRNA from either gene alone did not. This strongly indicates that suppression of the upstream gene does not inactivate the downstream gene and thus that the RNAi effect is post-transcriptional. The post-transcriptional effects of RNAi were directly observed using in situ hybridization to follow transcripts of genes suppressed by injection of dsRNA (Montgomery et al. 1998). There was a diminution of nuclear RNA from the suppressed gene as well as a total absence of the specific mRNA in the cytoplasm. This suggests that dsRNA establishes an intracellular state that destroys RNA transcribed and spliced from a specific gene. Both this study and other results are most easily explained if the specific RNA degradation occurs in both the nucleus and the cytoplasm.

dsRNA mediated suppression of specific gene expression has also been observed in plants. One demonstration of the phenomenon follows expression in plant cells of a recombinant RNA virus containing exonic sequences of an endogenous cellular gene. Expression of the cellular gene is suppressed in these cells when the recombinant viral RNAs are capable of replicating and not when they are replication incompetent (Angell and Baulcombe 1997). Viral RNA replication involves dsRNA. A similar phenomenon can be observed when a transgene is introduced into plant cells. The endogenous gene corresponding to the transgene can become suppressed (e.g., Vionnet et al. 1998), perhaps due to symmetric transcription of both strands of the transgene. Such symmetric transcription could arise by initiation in flanking sequences due to the presence of fortuitous promoter sites in plasmid DNA. The plant and nematode effects share the property of spreading. Examples of this are striking. Worms fed dsRNA exhibit a strong systemic interference phenotype (Timmons and Fire 1998) and introduction (into plants) of 500-bp fragments of a gene absorbed on the surface of a gold bead projectile can result in suppression of the gene in cells both immediately adjacent to the site penetrated by the bead as well as at very distant sites (Vionnet et al. 1998).

The purest demonstration that dsRNA mediates gene silencing in plants is the genetic study of Waterhouse et al. (1998). Transgenic plants were established which expressed either sense or antisense of a gene of the potato 
virus $\mathrm{Y}$ (PVY). Both transgenic lines of tobacco were susceptible to PVY infection. However, crosses of these tobacco lines that expressed transgenes for both the sense and antisense orientation and thus could generate dsRNA became resistant to PVY. This suggests that the two complementary RNAs transcribed from unlinked loci were able to anneal in the nucleus and induce a gene-specific suppressive state.

There are indications in C. elegans and direct evidence in plants that dsRNA may also act by generating a second mechanism, a silencing of transcription of a specific gene. In plants, the silenced gene has been shown to be hypermethylated, perhaps contributing to the inactive state (Wassenegger et al. 1994). In C. elegans, the only indication of such a gene-linked suppression is that the RNAi effect for some genes can be transmitted to the $F_{2}$ generation (Tabara et al. 1998). Silencing of an endogenous locus by transgenes has also been observed in Drosophila (see, e.g., Pal-Bhadra et al. 1997). In these cases, the copy number of the transgene appears to be important and there is no direct evidence that the silencing mechanism is mediated by dsRNA. One study strongly suggests that the mechanism of silencing is transcriptional, with the proteins of the polycomb complex becoming associated with the silenced endogenous locus (Pal-Bhadra et al. 1997). Polycomb complexes are known to be important for the silencing of genes during development. In the study, where dsRNA has been shown to be a potent and specific inhibitor of gene activity in Drosophila (Kennerdell and Carthew 1998), transmission through the germ line was not observed. In this case, the dsRNA was injected into the syncytial blastoderm embryos and it generated phenotypes in the L1 larvae but not in the progeny.

The finding that dsRNA may induce the transcriptional silencing of a specific gene could be important in several biological phenomena. For example, recent results suggest that the activity of an antisense promoter in the first intron of the gene for the mouse receptor for the insulin-like growth factor type-2 (Igf2r) is important for its paternal-specific repression (Wutz et al. 1997). In this case, dsRNA generated by symmetric transcription from the two opposing promoters might be a signal for establishing the allele-specific repression.

The cosuppression phenomenon and particularly its post-transcriptional gene silencing aspect (PTGS) in plants has been studied for a number of years. Several startling conclusions from these experiments may have implications for the RNAi effect in other organisms. First, that the PTGS effect, whether induced by a DNA segment or by a RNA duplex as part of the replicative intermediates of a RNA virus, is probably present in the cytoplasm as it inhibits specific gene expression from RNA viruses that are not known to enter the nucleus. Second, the gene-specific agents that stimulate or mediate the cosuppression effects are amplifiable by normal cells and will spread through plants by both plasmodesmatal and phloem channels (Vionnet et al. 1998). Furthermore, the agent(s) of suppression will pass through cells that do not contain any endogenous gene affected by the cosuppression effect. This suggests that the agent, which is likely to be composed of nucleic acids because of its gene-specific origin and effect, can be transmitted, and probably amplified, by cells utilizing the genetic information in the agent alone.

Evidence for amplification of the gene-specific agent comes from comparison of the amount of input dsRNA and the number of cells and number of mRNAs in those cells which are suppressed. For example, plant tissue with the PTGS state can be grafted onto other normal plants with a resulting spread of the PTGS state into the tissue of the grafted host (Vionnet et al. 1998). Similarly, a limited amount of dsRNA will generate RNAi in both the somatic tissue of the worm and the $F_{1}$ progeny (Fire et al. 1998). The only alternative explanation for the remarkable efficiency of the RNAi other than amplification would be the establishment of a highly catalytic RNA degradation process. It is possible, in fact likely, that both an amplification process as well as a catalytic process are part of the RNAi phenonemon in some organisms.

Restriction of infection by RNA viruses is almost certainly one of the biological consequences of the PTGS or RNAi state. Not surprisingly, some plant viruses appear to encode gene products which block the development of the PTGS state (Kasschau and Carrington 1998). The mechanism of suppression of the dsRNA induced genespecific silencing by the viral protein remains to be investigated.

The implications of a dsRNA-induced gene-specific silencing mechanism that is amplified by normal cells are astounding when considered in molecular terms. The effects discussed above can be diagrammed as follows:

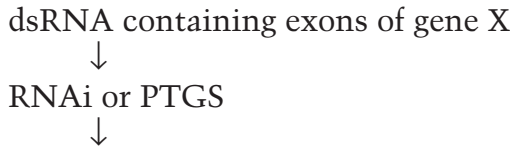

agent causes gene-specific degradation of mRNA from gene $X$ in both nucleus and cytoplasm; gene-specific agent, or inducing agent, can be duplicated by a normal cell and transmitted to other cells.

Speculation about the molecular processes, which underlie RNAi effects, might begin with a previously described mechanism for covalent modification of dsRNA (Bass and Weintraub 1988). Most eukaryotic cells contain one or more adenosine deaminases which convert many of the A residues in duplex RNA to inosine (I). The product RNA becomes sufficiently modified that the two strands of the RNA dissociate. This I-containing RNA is bound by proteins in extracts, so far not characterized, that are stable to electrophoresis in native complexes (Wagner and Nishikura 1988). One can speculate that this modified single strand RNA with bound proteins could be the agent, which interacts with nuclear and cytoplasmic RNAs, signaling their degradation. This agent might also be replicated by cellular polymerases, perhaps RNA polymerase II, which is thought to repli- 
cate viroid-type RNAs in both plant and vertebrate cells. If replicated by a base-pairing mechanism, the I residues would be converted to $G$ residues in the product. This type of conversion has been documented during the replication of RNA viruses where it is manifested as hypermutation of the viral genome (Bass 1997). Adenosine deamination of nuclear RNA has been characterized for sense and antisense RNAs from the early region of the DNA virus polyoma (Kumar and Carmichael 1997). In early-strand RNA recovered from late infected cells, approximately half of the adenosines were modified. Interestingly, this modified RNA was primarily confined to the nucleus and was apparently relatively stable.

In vertebrate systems, dsRNA was long ago recognized as a potent signaling molecule in the induction of interferons and execution of the antiviral state. Briefly, exposure of cells to dsRNA, such as poly(IC), potently induces the transcription of interferons that induce an antiviral state in cells. The antiviral state is characterized by the synthesis of a number of proteins that recognize dsRNA, a common property of the replication intermediates of RNA viruses. These proteins include a kinase, PKR which is activated by dsRNA, a $2{ }^{\prime}-5^{\prime}$-oligoadenylated synthetase activated by dsRNA and dsRNA-specific adenosine deaminase activities. The PKR kinase activity suppresses translation by phosphorylation of initiation factors and synthesis of oligo-2'-5' poly activates the endoribonuclease RNase L, which degrades RNA. However, neither this suppression of translation nor degradation of mRNA has been shown to be gene-specific in action. In fact, it remains a mystery how cells treated with interferon specifically suppress the translation of viral mRNAs in their cytoplasm and not cellular mRNAs. Perhaps some aspect of the RNAi effect occurs or can be induced in mammalian cells.

\section{References}

Angell, S.M. and D.C. Baulcombe. 1997. Consistent gene silencing in transgenic plants expressing a replicating potato virus X RNA. EMBO J. 16: 3675-3684.

Bass, B.L. 1997. RNA editing and hypermulation by adenosine deamination. Trends Biochem. Sci. 22: 157-162.

Bass, B.L. and H. Weintraub. 1988. An unwinding activity that covalently modifies its double-stranded RNA substrate. Cell 55: 1089-1098.

Fire, A., S. Xu, M.K. Montgomery, S.A. Kostas, S.E. Driver, and C.C. Mello. 1998. Potent and specific genetic interference by double-stranded RNA in Caenorhabditis elegans. Nature 391: 806-811.

Kasschau, K.D. and J.C. Carrington. 1998. A counterdefensive stategy of plant viruses: Suppression of posttranscriptional gene silencing. Cell 95: 461-470.

Kennerdell, J.R. and R.W. Carthew. 1998. Use of dsRNA-mediated genetic interference to demonstrate that frizzled and frizzled 2 act in the Wingless pathwy. Cell 95: 1017-1026.

Kumar, M. and G.G. Carmichael. 1997. Nuclear antisense RNA induces extensive adenosine modifications and nuclear retention of target transcripts. Proc. Natl. Acad. Sci. 94: 35423547.

Montgomery, M.K. and A. Fire. 1998. Double-stranded RNA as a mediator in sequence-specific genetic silencing and co-sup- pression. Trends Genet. 14: 255-258.

Montgomery, M.K., S. Xu, and A. Fire. 1998. RNA as a target of dsRNA-mediated genetic interference in Caenorhabditis elegans. Proc. Natl. Acad. Sci. 95: 15502-15507.

Ngo, H., C. Tschudi, K. Gull, and E. Ullu. 1998. Doublestranded RNA induces mRNA degradation in Trypanosoma brucei. Proc. Natl. Acad. Sci. 95: 14687-14692.

Pal-Bhadra, M., U. Bhadra, and J.A. Birchler. 1997. Cosuppression in Drosophila: Gene silencing of Alcohol dehydrogenase by white-Adh transgenes is polycomb dependent. Cell 90: 479-490.

Shi, Y. and C. Mello. 1998. A CBP/p300 homolog specifies multiple differentiation pathways in Caenorhabditis elegans. Genes \& Dev. 12: 943-955.

Tabara, H., A. Grishok, and C.C. Mello. 1998. RNAi in C. elegans: Soaking in the genome sequence. Science 282: 430431.

Timmons, L. and A. Fire. 1998. Specific interference by ingested dsRNA. Nature : 395, 854.

Vionnet, O., P. Vain, S. Angell, and D.C. Baulcombe. 1998. Systemic spread of sequence-specific transgene RNA degradation in plants is initiated by localized introduction of ectopic promoterless DNA. Cell 95: 177-187.

Wagner, R.W. and K. Nishikura. 1988. Cell cycle expression of RNA duplex unwindase activity in mammalian cells. Mol. Cell Biol. 8: 770-777.

Wassenegger, M., S. Heimes, L. Riedel, and H.L. Sänger. 1994. RNA-directed de novo methylation of genomic sequences in plants. Cell 76: 567-576.

Waterhouse, P.M., M.W. Graham, and M.-B. Wang. 1998. Virus resistance and gene silencing in plants can be induced by simultaneous expression of sense and antisense RNA. Proc. Natl. Acad. Sci. 95: 13959-13964.

Wutz, A., O.W. Smrzka, N. Schweifer, K. Schellander, E.F. Wagner, and D.P. Barlow. 1997. Imprinted expression of the Igf2r gene depends on an intronic CpG island. Nature 389: 745749 . 


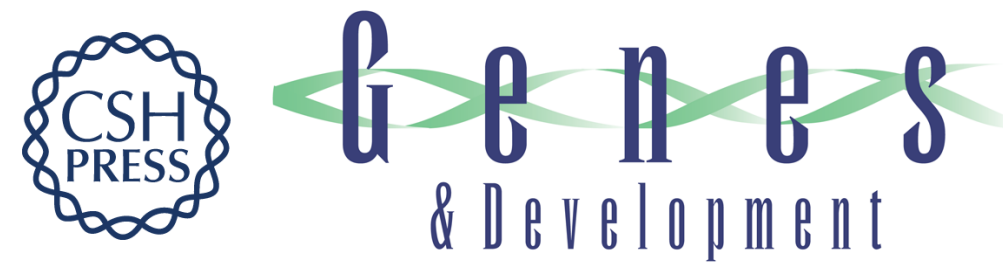

\section{RNAi and double-strand RNA}

Phillip A. Sharp

Genes Dev. 1999, 13:

References This article cites 19 articles, 8 of which can be accessed free at: http://genesdev.cshlp.org/content/13/2/139.full.html\#ref-list-1

License

Email Alerting

Receive free email alerts when new articles cite this article - sign up in the box at the top Service right corner of the article or click here.

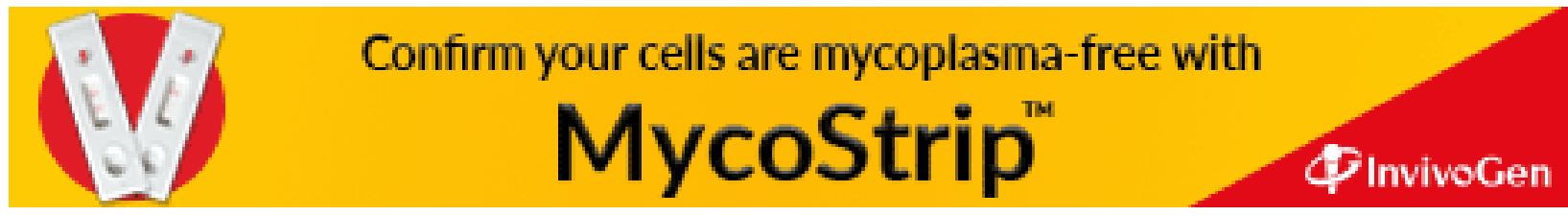

